\title{
Exploring the Touch and Motion Features in Game-Based Cognitive Assessments
}

\author{
JITTRAPOL INTARASIRISAWAT*, CHEE SIANG ANG ${ }^{\dagger}$, and CHRISTOS EFSTRATIOU, School \\ of Engineering and Digital Arts, University of Kent, United Kingdom \\ LUKE WILLIAM FEIDHLIM DICKENS, Department of Information Studies, University College London, \\ United Kingdom \\ RUPERT PAGE, Poole Hospital NHS Foundation Trust, United Kingdom
}

Early detection of cognitive decline is important for timely intervention and treatment strategies to prevent further deterioration or development of more severe cognitive impairment, as well as identify at risk individuals for research. In this paper, we explore the feasibility of using data collected from built-in sensors of mobile phone and gameplay performance in mobile-game-based cognitive assessments. Twenty-two healthy participants took part in the two-session experiment where they were asked to take a series of standard cognitive assessments followed by playing three popular mobile games in which user-game interaction data were passively collected. The results from bivariate analysis reveal correlations between our proposed features and scores obtained from paper-based cognitive assessments. Our results show that touch gestural interaction and device motion patterns can be used as supplementary features on mobile game-based cognitive measurement. This study provides initial evidence that game related metrics on existing off-the-shelf games have potential to be used as proxies for conventional cognitive measures, specifically for visuospatial function, visual search capability, mental flexibility, memory and attention.

CCS Concepts: • Human-centered computing $\rightarrow$ Touch screens; Haptic devices; Ubiquitous and mobile computing design and evaluation methods; • Applied computing $\rightarrow$ Health informatics.

Additional Key Words and Phrases: cognitive assessment, serious games, mobile health

ACM Reference Format:

Jittrapol Intarasirisawat, Chee Siang Ang, Christos Efstratiou, Luke William Feidhlim Dickens, and Rupert Page. 2019. Exploring the Touch and Motion Features in Game-Based Cognitive Assessments. 1, 1 (September 2019), 25 pages. https: //doi.org/10.1145/nnnnnnn.nnnnnnn

\section{INTRODUCTION}

Cognitive impairment occurs in a wide range of clinical conditions which adversely impact higher brain function. Causes of cognitive deficits include stroke, dementia, traumatic brain injury, and alcohol and drug use as well

${ }^{*}$ This is the first author
${ }^{\dagger}$ This is the corresponding author

Authors' addresses: Jittrapol Intarasirisawat, ji81@kent.ac.uk; Chee Siang Ang, c.s.ang@kent.ac.uk; Christos Efstratiou, c.efstratiou@kent.ac. uk, School of Engineering and Digital Arts, University of Kent, Jennison Building, Canterbury, Kent, CT2 7NT, United Kingdom; Luke William Feidhlim Dickens, Department of Information Studies, University College London, Gower Street, London, WC1E 6BT, United Kingdom, 1.dickens@ucl.ac.uk; Rupert Page, Poole Hospital NHS Foundation Trust, Poole, Poole, BH15 2JB, United Kingdom, rupert.page@poole.nhs.uk.

Permission to make digital or hard copies of all or part of this work for personal or classroom use is granted without fee provided that copies are not made or distributed for profit or commercial advantage and that copies bear this notice and the full citation on the first page. Copyrights for components of this work owned by others than ACM must be honored. Abstracting with credit is permitted. To copy otherwise, or republish, to post on servers or to redistribute to lists, requires prior specific permission and/or a fee. Request permissions from permissions@acm.org.

(c) 2019 Association for Computing Machinery.

XXXX-XXXX/2019/9-ART \$15.00

https://doi.org/10.1145/nnnnnnn.nnnnnnn

, Vol. 1, No. 1, Article . Publication date: September 2019. 
as less common causes such as developmental disorders and encephalitis [2, 18, 34]. Often older people with mild cognitive impairment are at higher risk of progressing to more severe cognitive impairment (e.g. dementia) that interferes significantly with individuals' daily life [31]. Early detection of subtle signs of cognitive decline provides a greater opportunity for timely intervention [9]. Similarly, athletes with exposure to accumulative traumatic brain injuries in sports, e.g. boxing, football and rugby require regular monitoring of cognitive changes to determine whether they can safely resume their participation in the game. In sport settings, the assessments typically need to be conducted on-site with short administration time [7]. In addition, there is an underlying need for cognitive measures that allow frequent repetitive testing. However, owing to the lack of multiple variations of the tests and the ability to self-administer, the current paper-based approach is not feasible for frequent repetitive measurement.

To overcome these limitations, a growing body of research has examined the feasibility of assessing cognitive functions on digital platforms. A number of studies have investigated the use of computerised applications for cognitive screening, citing the capacity to generate random stimuli and capture highly accurate in task measures of performance as key advantages [7, 23, 30, 39, 53]. However, the potential lack of motivation to carry out boring and repetitive tasks could discourage users from taking frequent assessments as needed to monitor their condition continuously [10]. We believe that the entertaining nature of game-based assessments can better motivate users to maintain interest during the test and promote engagement with frequent assessments over time $[10,26,27,47]$. Existing literature has so far only examined the feasibility of using gameplay performance as a means for cognitive assessment. To our knowledge, there has not been any attempt to explore gameplay behaviour as revealed through patterns of tap and swipe interactions on the screen, hereafter called touch interactions and patterns of gyroscope and accelerometer sensor readings, hereafter called device motions by a player, as tools for cognitive assessment.

The overarching aim of this study, therefore, is to explore if user-game interaction behaviour can be used to infer cognitive abilities with the focus on touch gestures and device motions in addition to gameplay performance. Specifically, we investigate correlations between features from gaming behaviour and cognitive performance. Previous studies have shown that engagement with the games is the key factor for sustaining attention in continuous assessments $[10,26,27,47]$. Therefore, in this study, we employed a broader set of games with a strong track record of being interactive and engaging to identify potential candidates among existing popular games. Moreover, we attempt to identify key common attributes between varying styles of gameplay that may act as proxy markers for specific cognitive functions. Our findings inform our recommendations on game interaction design to capitalise on the use of touch interaction and device motion data in game-based cognitive assessments.

\section{RELATED WORK}

In healthcare, a multitude of neuropsychological tests have been developed and used by clinicians for assessing cognition. Mini Mental State Examination (MMSE) [46], Montreal Cognitive Assessment (MoCA) [29] and Addenbrooke's Cognitive Examination-III (ACE-III) [15] are widely used for cognitive impairment screening and monitoring in clinical settings. Currently, these assessment tools are typically paper-based and not designed for self-administration [15]. Thus, it is infeasible to run the tests frequently to monitor changes in cognitive functions over time due to learning effects [46], costs and resource requirements around availability of qualified clinical staff to administer them. These can adversely affect the ability of clinicians to detect the early signs of decline in cognitive functions, potentially delaying diagnosis and treatment as well as undermining the effectiveness of medication or other interventions [37]. These limitations have led to a call for alternative approaches. Taking advantage of computerised assessments may provide more precise test results, better control of stimulus presentation and ease of administration to assess cognitive abilities. One of the most widely used computerised tools for cognitive measurements in clinical research is the Cambridge Neuropsychological Test 
Automated Battery (CANTAB). The CANTAB system includes various tests measuring a range of cognitive functions, e.g. executive function, attention, memory and decision making [55]. It has been extensively used to assess cognitive functions in older people [32], athletes with exposure to repeated brain injuries [7], for paediatric neuropsychological assessment [25], HIV dementia patients [34] and alcohol drinkers [14]. However, trained clinical personnel are required for protocol administration of CANTAB.

In recent years, there has been a growing interest in developing mobile applications for cognitive assessment and diagnosis of cognitive impairment. Touch-based devices are now relatively inexpensive and easily accessible. Unlike the use of a keyboard and mouse, indicated as a barrier to use for some older adults [42], intuitive touch controls reportedly enhance user-application interaction and ease of use [6]. By adapting common tasks in standardised paper-based tests, several mobile applications were specifically developed to be cognitive impairment screening tools [3, 30, 53]. A mobile cognitive screening application (MCS) [53] employed a battery of tests measuring a number of cognitive functions, e.g. arithmetic, attention and executive functions on a tablet device. Their analysis demonstrates strong correlations between MCS test results and MoCA in older adults and its feasibility for cognitive screening of dementia. In a larger study, test results from the proposed cognitive screening tool CADi [30] were strongly correlated with MMSE scores. CADi also showed high sensitivity and specificity in differentiating people with dementia from healthy controls. These studies showed evidence that mobile applications can discriminate healthy individuals from cognitively impaired patients. Nonetheless, due to the repetitive nature of such applications, it is challenging to motivate users to engage in frequent administrations to monitor cognitive changes over time.

"Serious games" have recently gained increasing research attention for their potential to improve sustained participation in continual assessment and therapy by incorporating elements of fun and user engagement in their design [10, 11, 26, 27, 47]. Serious games are those designed for some additional purpose beyond pure entertainment, such as: training, marketing, communicating, assessing and/or enhancing cognitive and physical health [33]. For instance, a tabletop-gaming platform in the Eldergames project was developed to improve cognitive functions in older adults. Their findings showed that their interactive tabletop games were wellaccepted with regard to usability and reported to create a positive experience [11]. However, this approach requires space for equipment setup and is thus not feasible for running in a large-scale experiment. In contrast, the ubiquitous computing power of modern mobile devices offers promising solutions for data collection and processing for cognitive assessment and monitoring outside clinical settings. Mobile versions of serious games have been developed to simulate common daily activities such as cooking [27] and supermarket shopping [54], in order to assess and help improve cognitive functions among people with mild cognitive impairment (MCI). To complete tasks in the game scenarios, a multitude of cognitive processes were involved, e.g. object recognition, attention, visual search, memory and executive functions. By comparing in-game task performance and classic cognitive assessments, e.g. MMSE and TMT [45], their findings demonstrated significant correlations between variables in the games and results from standard cognitive measures. Unlike the simulation-based designs that artificially represent real-world scenarios in such games, replicating a popular casual game Whack-a-Mole presents more game-like attributes and reduces the feelings of being tested [47]. A Go/No-Go discrimination task has also been incorporated into serious games to measure cognitive inhibition. The significant correlations between median response time and cognitive test scores suggested that this in-game feature could be used as a predictor for cognitive status. A recent systematic review found that the use of gamified tasks can help improve drop-out rates in longitudinal studies including a reduction in test anxiety [26]. Because of their entertaining nature, these game-based assessments were reportedly well received by the users even in older adults. In spite of common misperceptions, a systematic review reported that older adults enjoyed video games and benefited from game-based cognitive intervention [20]. This was supported by a recent report demonstrating that 23 per cent of the U.S. gamers were 50 years and older [41]. Hence, these studies have emphasised the potential of 
serious games as highly engaging cognitive assessments to monitor changes in cognition outside of a clinical environment for populations with cognitive disorder across age groups.

As well as supporting precise data collection of in-game behaviours and performance, modern mobile devices are capable of sensing user-game interaction behaviour via built-in sensors such as accelerometers, gyroscope and magnetometer. A number of existing studies exploited these sensing capabilities to passively collect data about a user's interaction with their phone, their movement and surroundings in order to infer users' affective states [12] and health conditions [1,35]. Gao et al. [12] exploited touch gestural patterns during gameplay to predict the self-reported emotional state of participants from a 4-class task, and achieved up to $77 \%$ accuracy. A more recent study combined built-in mobile sensors with mobile phone usage and physiological signals for automatic discrimination of stress condition with above $75 \%$ classification accuracy [35]. In a study involving children with autism [1], researchers showed that gesture and movement patterns from iPad gameplay can differentiate children with autism from typically developing children with $93 \%$ accuracy. Their study also validated the use of game-based assessments to enhance engagement and attention for experimental tasks involving children. Previous studies have shown that game-based assessments offer potential advantages over traditional cognitive tests. In addition to replayability, the game-based approach allows self-administration with more accurate measurement and increases engagement in continual assessments.

Mobile gameplay mainly involves touch gestures that users perform on the screen. In order to discover whether such gestures can be related to cognitive performance, we explored a broader range of studies where hand movement has been shown to be related to cognitive abilities. Most of the existing literature in that domain focuses on people with diagnosed neurological disorders. Previous studies have shown that changes in fine motor abilities are commonly observed in patients with Alzheimer's disease, mild cognitive impairment [36], schizophrenia [44] and obsessive-compulsive disorder (OCD) [28].

A series of comparative studies $[28,36,44]$ investigated speed, quality and accuracy of kinematic handwriting movement using a digitising tablet in samples of various cognitively impaired patients. Significant impairment in the regularity of repetitive hand movement was detected through hand-motion parameters. Patients with Schizophrenia differed from healthy controls in automation parameters (mean peak acceleration and number of direction changes of velocity (NCV)) and regularity of stroke motion parameters (standard deviations of velocity, acceleration and stroke duration) [44]. Similarly, patients with Alzheimer's disease and mild cognitive impairment exhibited a lower degree of automation of hand movement in drawing repetitive circles than healthy controls. A significant negative correlation was also found between MMSE and NCV [36]. Mean stroke length in the writing task appeared to be shorter in patients with OCD than in healthy controls [28]. Moreover, due to age-related cognitive declines, older adults were found to exhibit fine motor disturbance, for instance, slower velocity and higher variability in movement [19]. These studies demonstrate that a higher degree of movement variability is correlated with declines in cognitive performance.

It is important to note that all these studies have explored hand movement in non-time-dependent tasks, such as handwriting. However, gameplay hand movement is closely related to user reactions on game stimuli. The characteristics of touch gestures in games are highly dependent on the time the user perceives stimuli in the game and the limited time they have to perform a specific gesture. Therefore the shape, speed and length of a gesture can be different, depending on the time it takes to perceive a game trigger. For example, a slow response time in identifying a game object that a player needs to interact with (e.g. in "Fruit Ninja" spotting a fruit that is about to move out of the screen) could result in a faster and more erratic gesture in order to complete the gesture in the reduced time available. Previous studies have shown that mental fatigue [21] and age [8] adversely affect the speed of processing resulting in slower reaction time. This means that in certain games, faster and more erratic gestures could be an indicator of slower response time to visual stimuli, and therefore indicative of cognitive decline. 


\section{RESEARCH QUESTIONS}

Existing work has demonstrated a potential relationship between cognitive performance and hand movement/gestures. In order to explore how these observations are reflected in the domain of smartphone games, we formulate a set of research questions. In particular, we intend to explore the extent to which the shape and timing of gestures could be related to changes in cognitive abilities.

RQ1: Are the swipe length and shape of touch gestures related to changes in cognitive performance? Considering the related work in writing exercises, there are strong indications that changes in cognitive performance could be related to changes in the length and shape of hand movements. Although gameplay touch interactions are different in nature compared to those of handwriting - gestures are time-dependent, and triggered by other stimuli - we anticipate that the nature of the gesture shape could demonstrate statistically significant correlations with different cognitive abilities.

RQ2: Is the speed of touch gestures related to changes in cognitive performance? Speed in game interaction is mainly related to responses to visual stimuli. In that respect, the temporal characteristics of gestures would be related to the time needed for a person to respond to such stimuli. Previous work has established that reduced cognitive performance is linked to increased response time to stimuli. We want to explore how such an increase in response time can be related to the timing characteristics of touch gestures.

RQ3: Are the characteristics of the physical movement of the mobile device related to changes in cognitive performance? We consider that in smartphone gameplay, the device movements are predominantly influenced by the user's touch gestures as they interact with the game. Assuming that RQ1 and RQ2 have revealed correlations between touch gestures and cognitive performance, we further want to explore if device movements, as captured by the smartphone's sensors, are also be correlated with changes in cognitive performance.

In order to answer these research questions, we conducted a controlled study to capture gameplay information from a range of users and analyse them with respect to differences in cognitive abilities.

\section{MATERIALS AND METHODS}

We conducted a study where participants were asked to play games to assess their cognitive performance. The objective of the study was to find links between how they use touch gestures in popular games and their cognitive performance as measured through established cognitive function tests.

\subsection{Participants}

In this study, 22 healthy participants between the ages of 18-34 years were recruited through email invitations for voluntary participation in the study via our university mail lists. Volunteers were offered a $£ 20$ gift voucher as an incentive for taking part in the study. Potential participants were excluded if they were critically ill, diagnosed with neurological or psychiatric disorders, diagnosed with Parkinson's disease or arthritis, currently receiving psychoactive medication, blind or colour-blind, unable to understand verbal English instruction or considered to be excessive video gamers (with a playing time of more than 3 hours a day).

Initially, 10 females and 12 males were recruited. After reviewing all collected data, 1 participant was later excluded from the analysis for accidentally resetting a game and so was unable to continuously play the game over the given time period. The final data set hence consisted of 21 participants ( 9 females and 12 males). 10 of whom use English as their first language. All participants are right-handed. Participants reported that recently they only played mobile games at most 2-3 days a week with $71 \%$ saying that their average gaming session time was less than an hour, while $24 \%$ and 5\% played average sessions of 1-2 hours and 2-3 hours respectively.

This study was approved by the Research Ethics Advisory Group of the School of Engineering and Digital Arts, University of Kent, UK (Ref. No. 0721617). All participants provided written informed consent after a complete description of the study. 


\subsection{Data Collection Procedure}

In our study, we only included cognitively normal individuals without any diagnosed conditions. Under normal conditions, measuring the cognitive abilities of such individuals using standardised cognitive assessment instruments would typically exhibit scores within a narrow range closer to the maximum possible scores [36]. Considering that the possible low variation in cognitive scores within our subjects could limit the extent of the correlation analysis findings, we decided to introduce in our study tasks that would induce a temporary decline in cognitive performance. Specifically, the study was carried out in two sessions on two separate days. In one of the sessions, a selected mental fatigue task was used to stimulate mental exhaustion, which induced a decline in participants' cognitive performance. The session with the intervention was solely introduced in our experimental design with the particular aim to improve data variability in paper-based cognitive assessment scores. Hence, data from the two sessions were combined in the subsequent analysis. The experiment was conducted individually in a quiet room. All participants played two sessions two weeks apart, with one session starting with the mental fatigue task. Block randomisation was used for session type, splitting participants into two groups A and B. Groups were therefore roughly balanced in terms of gender and English as a first language, with group A experiencing the mental fatigue task in session 1. In order to reduce the effect of prior game experience in the study, in their first session, participants received instructions on how to play the games and were asked to play each game for 10 minutes to familiarise themselves with the gameplay. Participants were instructed to play the games while being seated on a chair without an armrest. They were advised to hold the device with one hand and play the game with another hand. Only one finger was allowed to touch the screen at a time. The justification for asking players to play two-handed is that playing games with a single hand would make it difficult to access certain screen areas, e.g. top left corner. Moreover, with single hand playing, hand size and finger length are likely to influence the stroke patterns and this would therefore potentially introduce confounding factors leading to spurious associations.

4.2.1 Basic Session. All participants were asked to complete a mood questionnaire (Hospital Anxiety and Depression Scale - HADS [52]) and submit a sleep diary prior to commencing the session. Participants were next required to perform a set of cognitive measures (see later) and then to play three games in succession, 10 minutes per game, without breaks. All games were pre-installed on a Samsung S6 device, without a screen protector. The sequence of the games was also randomised to avoid order effects.

4.2.2 Mental Fatigue-inducing Task Session. In this session, participants followed the same steps described in the basic session, except that they were required to perform a mental fatigue-inducing task for 30 minutes prior to taking a series of cognitive ability tests. To induce cognitive overload, a 2-back test was used, in which participants were continually presented with a series of letters and instructed to respond as quickly and correctly as possible when the displayed letter was the same as the one that had appeared 2 presentations ago [38]. The aim of the mental fatigue-inducing task was to induce a broader range of cognitive ability scores for our participants. A wider range in cognitive abilities across the study could help highlight potential correlations more clearly.

\subsection{Games}

The three games used in the study were: Tetris, Fruit Ninja and Candy Crush Saga. Games were selected based on: being easy to learn, being highly engaging for most players, and involving intensive touch interactions (rich in data). The three games were chosen together for a diversity of gameplay characteristics in order to explore different demands on cognitive function, i.e. visuospatial in Tetris [22], response inhibition and attention in Fruit Ninja [24] and visual search in Candy Crush Saga.

In order to passively collect interaction data on touch, sensor and gameplay, we developed our own versions of these three games based on the available assets [48-50] in Unity3D asset store. Several modifications were 


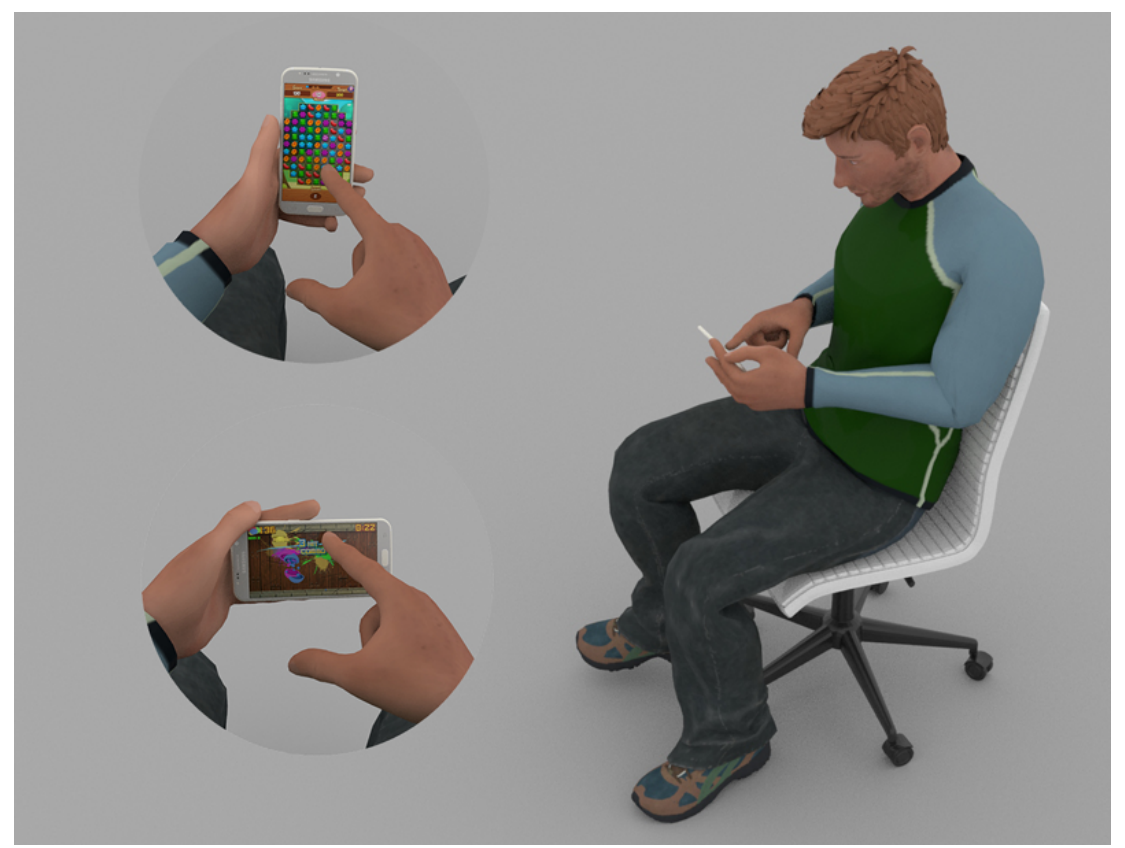

Fig. 1. A graphic displaying the body positioning, finger placement and hand grasp participants were advised to adopt during touchscreen interaction while playing the games.

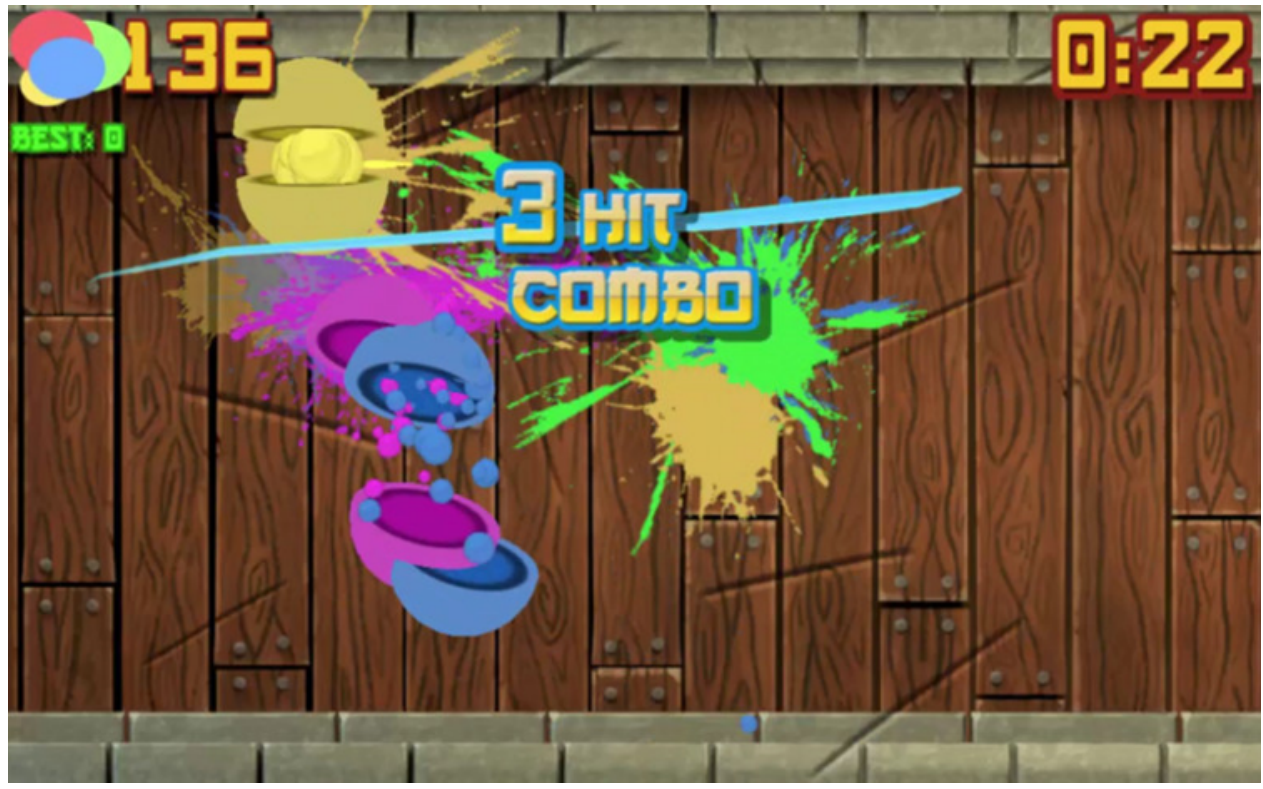

Fig. 2. A screenshot of a variation of Fruit Ninja in Unity3D asset store. To win points, players have to slice fruit thrown in the air with a blade using their fingers to swipe across the screen. The game is over when three pieces of fruit are missed. 


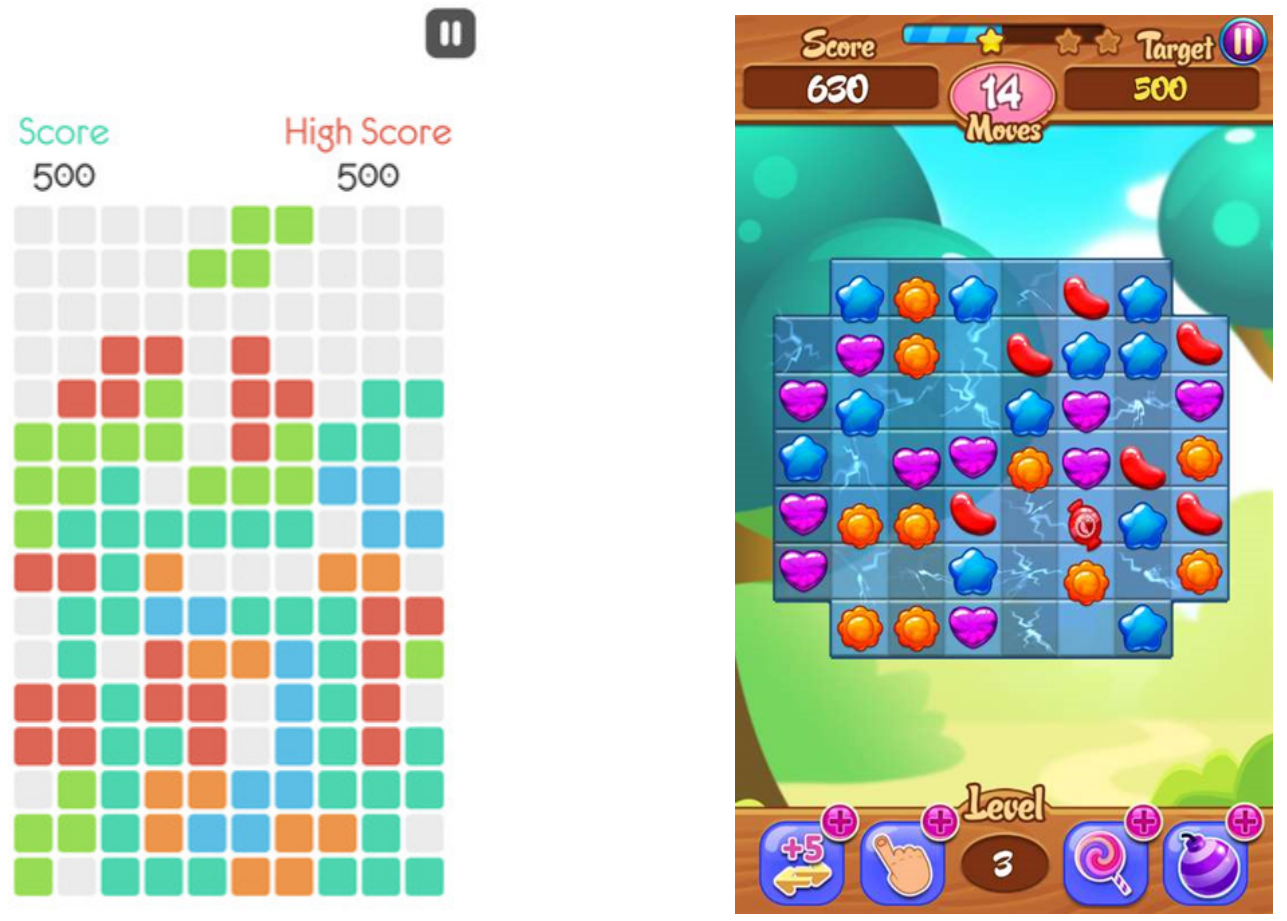

Fig. 3. (Left) A screenshot of a variation of Tetris in Unity3D asset store. In the game, players are required to manipulate the falling shape with the goal to complete a 10-block horizontal line without gaps. Swiping left/right is to move the shape to the left/right one block unit at a time while swiping down is to force it to fall into the stack instantly. Tapping the screen is to rotate it by 90 degrees. (Right) A screenshot of a variation of Candy Crush Saga in Unity3D asset store. In this puzzle match-3 game, players have to swipe the screen to swap two adjacent candies vertically or horizontally to match coloured candies in a combination of three or more to earn points.

implemented to allow the games to keep track of touch interactions and physical motions through built-in sensors including gameplay activities. In order to precisely capture their hand movements, participants were instructed to hold the phone in their hand and not to place it on the table while playing. To simplify touch interactions, games were modified to support only single touch events (one finger on the screen at a time).

\subsection{Measures}

Global cognitive functioning was measured using ACE-III, a cognitive screening instrument used in assessment of attention, memory, language and visuospatial function [15]. In this study, we only used results from ACE-III subtasks to measure cognitive abilities in attention, memory and visuospatial domains. Higher values indicate increased cognitive performance and vice versa.

Attention (ATN) refers to the participants' ability to stay focused on target stimuli or tasks over a long period of time. We anticipated ATN having a significant influence on performance in all three games.

Memory (MEM) is the ability to maintain information over time. Working memory is usually engaged when performing spatial and visual tasks to recall information that has been recently perceived. Demands on this particular cognitive function are most relevant to the game of Tetris as players are required to remember the 
current stack of shapes in order to decide where particular shapes may fit to clear a number of rows. We did not expect Candy Crush and Fruit Ninja to place particular demands on memory.

Visuospatial ability (VISP) refers to the ability to understand spatial relationships between objects. We anticipated VISP cognitive function to be most relevant to Tetris as the ability to identify the relationship between the falling shape and the current stack is key to performance. Fruit Ninja places slightly different demands on VISP function namely on how to determine the distance between falling items and the bottom edge of the screen. We did not expect Candy Crush to place particular demands on VISP function.

Trail Making Test Part A and B (TMTA and TMTB respectively) were used to measure participants' visual search, processing speed including visual attention. However, in addition to the previous cognitive domains, TMTB also tests participants' mental flexibility [45]. Longer time spent to complete TMT tasks indicates a decline in cognitive performance and vice versa. Both measures were regards as relevant to all three games.

Response Inhibition (RESIN) was measured by the Stroop Colour-Word test [15, 17]. Higher values indicate a decline in cognitive performance and vice versa. We anticipated that RESIN is most relevant to Fruit Ninja in that participants must inhibit their responses to bombs (no-go stimuli) and Candy Crush as participants should not make a random move or the first match they see but rather make a deliberate move strategically to win more points.

Therefore, in this present work, we investigated associations in different sets of cognitive domains specific to each particular game as described above and listed in Table 4.

\section{DATA PROCESSING}

\subsection{Outlier Removal}

Prior to our analysis, we examined the score distribution of each cognitive task in order to detect if there were unusually large or small values among all observations. Using the standard deviation method, the observations with values greater than 3 standard deviations above or below the mean are considered outliers.

Based on this exclusion criterion, participant 1 and 8 were excluded from the TMT-A task and participant 8 from the TMT-B task. When examining closer to identify reasons, participant performance on tasks where they appeared as outliers was found to coincide with unusual events during the experiment, including a participant restarting a task without the clock being restarted and a participant reporting being distracted from the task by a procedural error.

\subsection{Cognitive Scores}

As the mental fatigue-inducing task was introduced into the study to gain greater variability in cognitive assessment scores, we examined data variability in terms of the distribution of normalised cognitive assessment scores in both sessions using mean, interquartile range (IQR) and stand deviation (SD). In the session with the mental fatigue-inducing task, we found noticeably higher degrees of variation in attention (IQR increased from 0.17 to 0.25 , SD increased from 0.15 to 0.24 and mean declined from 0.91 to 0.81 ) and TMT-A (IQR increased from 0.30 to 0.33 while SD and mean remained roughly the same. Similarly, in TMT-B the mean increased from 0.27 to 0.33 . Though, IQR and SD remained relatively the same.

\subsection{Features}

5.3.1 Touch Data. In this initial exploration, our aim was to assess the relationship between touch interaction dynamics and cognitive scores. For each gestural touch interaction, we recorded a series of coordinates of the finger contact areas on the screen with timestamps. To identify possible touch feature patterns, quiver plots in Figure 4 were used for visual inspection to display each corresponding pair of data points as a vector with an arrowhead. In particular, the swipe gestures made throughout the entire gameplay were classified into four 
directions, i.e. up, right, down and left based on the suggestion proposed by Bevan et al. [4] that directions of swipes affect the characteristics of swipe gestures. Importantly, features were measured in different directions because we believe that different game mechanics can potentially influence gestural characteristics in particular directions. For instance, in Tetris, swiping left or right is to move the falling shape to the corresponding direction one step at a time regardless of the performed swipe length. In contrast, players are likely to swipe their finger down to drop the piece as quickly as possible when they feel confident of the target location. Therefore, we expected a lower number of longer and faster downward swipes as compared to horizontal swipes. Furthermore, it was reported in the neuroscientific literature that hemispheric utilisation bias demonstrates a strong influence on visuospatial attention. Particularly, individuals with a left hemisphere (LH) utilisation bias exhibited difficulty in selectively attending to stimuli presented on the left visual field [40]. Since participants are all right-handers who commonly demonstrate left-hemisphere dominance [43], these right-handed individuals were expected to display attentional bias to in-game stimuli appearing on the right. This led us to anticipate differences in gestural characteristics between leftward and rightward directions.

Different colours were used to indicate swipe directions in the plots. Swipe direction was determined using a swipe angle as illustrated in Figure 5. A full circle, which represented 360 degrees in one full rotation, was split into four 90 degree quadrants. A swipe was labelled with the corresponding direction of the quadrant in which the swipe angle landed. From the visual inspection of the touch patterns, in certain plots, we spotted patterns that seemed to be unusual as several swipes shared the same data points in the lower left area of the plots. We believed that these touch data points were mistakenly captured when participants' palm touched the screen by chance while holding the phone in their hand. These faulty records were automatically identified by thresholding the distance between two data points within a given swipe and then removed from further analysis. Four measures, specifically, total number, length, speed and directness index were used to extract an initial set of features across the four directions of swipes including taps. It is worth pointing out that directness index is a feature to quantify the "straightness" of a swipe. If a swipe is carried out in a perfectly straight line, the directness index will be one whereas the value will be greater than one in a curved swipe. The features were extracted and computed per session based on the functions denoted in Table 1.

Due to differences in gameplay styles, participants had a tendency to perform distinct touch gesture patterns in each game. Different subsets of the original touch-based features were chosen from the full table for each game.

Tetris. Horizontal swipes move blocks to the left or right and tapping the screen rotates the blocks by 90 degrees. Swiping downward forces the blocks to fall into the bottom stack instantly. This could indicate confidence in the location where a falling block will fall with its current orientation. As upward swipes have no function in the game, all features in the upward direction were excluded. Performed swipes exhibited a low degree of variability in directness indexes $(\mathrm{M}=1.04, \mathrm{SD}=.10)$. This result indicates that the game mechanics were in favour of straight swipes. Therefore, features associated with directness index were excluded accordingly.

Fruit Ninja. Looking over the touch plots, the swipe gestures in Fruit Ninja were clearly more idiosyncratic than in other games. Most swipes were drawn continuously in erratic zigzag patterns. As a result, the swipe length increased significantly in contrast to the number of swipes, which considerably declined. As the number of swipes and taps were not much different between participants, these features were excluded. Owing to the arbitrary zigzag move, a single swipe was largely performed in multiple directions. It was impossible to label a swipe with a single direction. Instead of extracting gesture features by direction, it made more sense to extract features on all swipe gestures together (irrespective of direction). Performed swipes exhibited a great degree of variability in directness indexes $(M=25.48, S D=35.11)$ than in other games. The DI feature was, thus, included.

Candy Crush. Unlike Tetris tapping the screen does nothing in Candy Crush. Thus, the number of taps was not included in our feature list. Performed swipes exhibited a low degree of variability in directness indexes $(M=1.07$, 

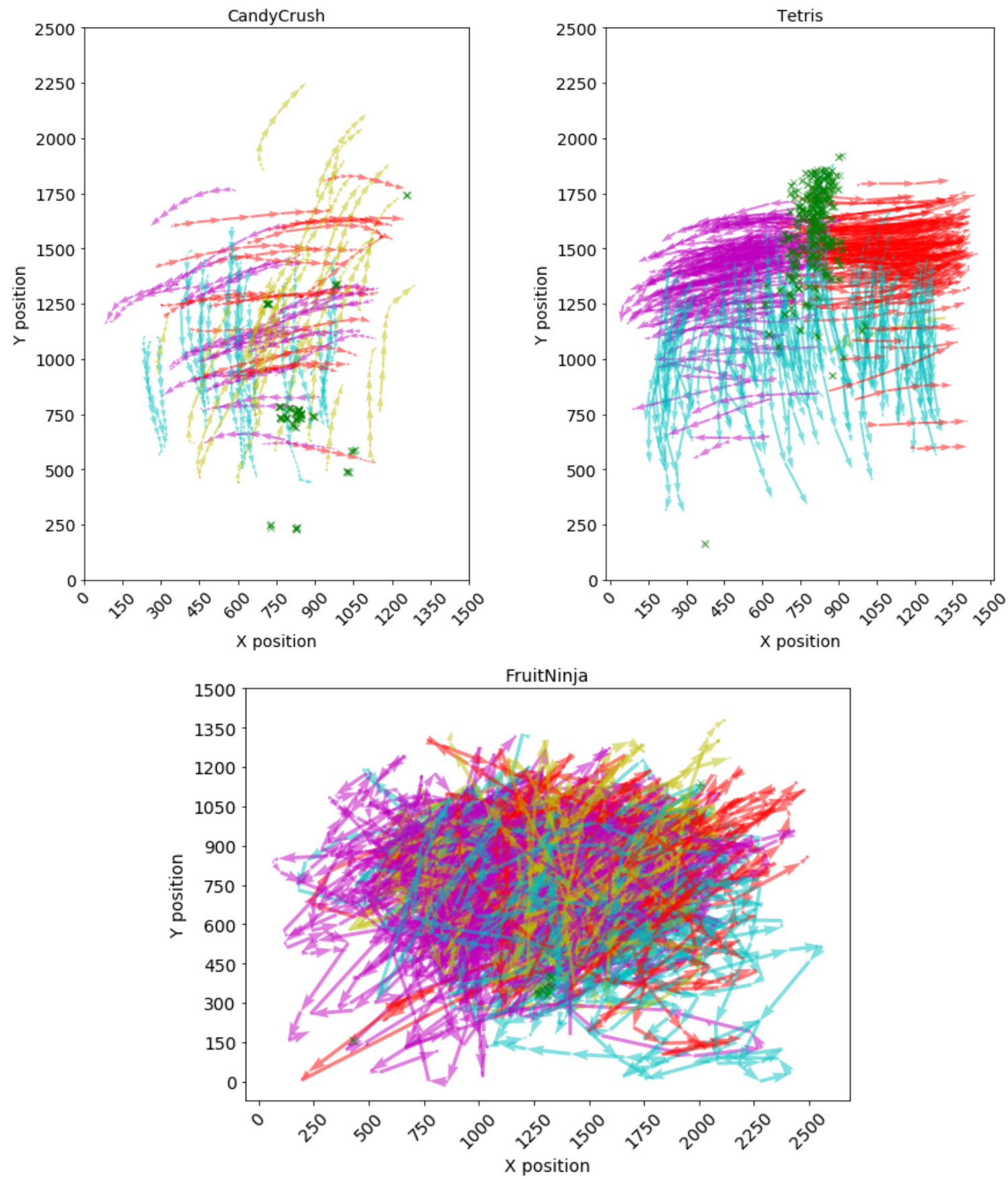

Fig. 4. Touch patterns of an individual participant in each game. Each swipe renders a line with arrowheads by connecting all data points within that individual interaction. Lines are drawn in different colours to represent the directions of swipes. Coordinates of tap interactions are plotted with ' $X$ ' markers. Values in the horizontal and vertical axes represent touch points on Samsung S6 screen with the display resolution of 1440 x 2560 pixels. 


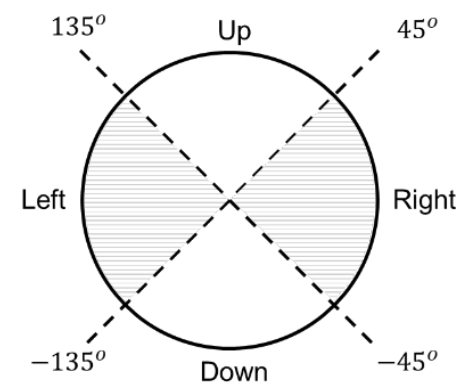

Fig. 5. The four quadrants defining the general direction of a swipe.

Table 1. A full table of initial touch features.

\begin{tabular}{lc}
\hline Feature & Function \\
\hline Total Number of Swipes by Direction* & $N$ \\
Total Number of Taps & $N$ \\
Mean Length of Swipes by Direction* & $\bar{l}=\frac{\sum_{i=1}^{N} l_{i}}{N}$, where \\
Mean Speed of Swipes by Direction & $\begin{array}{c}l_{i}^{*}=\sum_{j=1}^{n_{i}-1} \sqrt{\left(x_{i,(j+1)}-x_{i, j}\right)^{2}+\left(y_{i,(j+1)}-y_{i, j}\right)^{2}} \\
n_{i} \text { is the number of data points in the } i^{t h} \text { swipe interaction } \\
\bar{v}=\frac{\sum_{i=1}^{N} v_{i}}{N}, \text { where }\end{array}$ \\
$v_{i,}=\frac{\left(l_{i,(j+1)}-l_{i, j}\right)}{\left(t_{i,(j+1)}-t_{i, j}\right.}$ and
\end{tabular}

${ }^{*} \mathrm{Up}$, down, right, and left

$\mathrm{SD}=.11)$. This result indicates that the game mechanics were in favour of straight swipes. Therefore, features associated with directness index were excluded accordingly.

Touch features included in the analysis for each game are listed in Table 2.

5.3.2 Sensor Data. In order to allow us to explore if there is any motion pattern associated with cognitive performance, device motions during gameplay were captured using the built-in 3D accelerometer and gyroscope. The accelerometer offers acceleration measurements in three dimensions. These measurements include the gravity accelerations which can help identify the orientation of the device. Values are captured in the range of $(-1 \mathrm{~g},+1 \mathrm{~g})$ for each axis.

Rotational motion was captured using the gyroscope. The speed of rotation around each axis is measured in radians per second. When the phone is rotated counter-clockwise, the rotation will be positive. By contrast, the rotation will be negative when turning the phone in a clockwise direction. 
Table 2. Extracted touch features for each game.

\begin{tabular}{lccc}
\hline Feature & Tetris & Fruit Ninja & Candy Crush \\
\hline Total Number of Swipes by Direction* & $\checkmark$ & & $\checkmark$ \\
Total Number of Swipes in Horizontal or Vertical & $\checkmark$ & & $\checkmark$ \\
Total Number of Swipes & $\checkmark$ & & $\checkmark$ \\
Total Number of Taps & $\checkmark$ & & $\checkmark$ \\
Mean Length of Swipes by Direction* & $\checkmark$ & & $\checkmark$ \\
Mean Length of Swipes in Horizontal or Vertical & $\checkmark$ & & $\checkmark$ \\
Mean Length of Swipes & $\checkmark$ & $\checkmark$ & $\checkmark$ \\
Mean Speed of Swipes by Direction* & $\checkmark$ & & $\checkmark$ \\
Mean Speed of Swipes in Horizontal or Vertical & $\checkmark$ & & $\checkmark$ \\
Mean Speed of Swipes & $\checkmark$ & $\checkmark$ & \\
Mean Directness Index of Swipes & & $\checkmark$ & \\
\hline
\end{tabular}

${ }^{*}$ Right, left and down in Tetris and all four directions in Candy Crush

In addition to raw values from the three axes, we also included the magnitude of a 3D vector as a basic attribute for feature extraction. We are interested in physical movement rather than orientation. Therefore, we use the standard deviation of the eight basic attributes (four from each sensor) as features to describe variation in physical movement. The mean and the sum of the acceleration and speed of rotation magnitude are also included. As a result, twelve features were extracted from sensor data per session for each game.

5.3.3 Gameplay Data. Although it is not part of our main research questions, we also explored associations between gameplay patterns and cognitive performance, based on the significant findings from a number of related studies [27, 47,54]. Despite of sharing the same characteristics of being highly engaging and easy to play, each game has its own gameplay and rules which is very much distinct from others. Therefore, different sets of gameplay features were extracted per session from each game as listed in Table 3.

\section{RESULTS}

A total of 42 samples were collected from 21 participants in the two-session experiment. Prior to analysis, 2 samples from participant 1 were identified as outliers in measuring TMT-B, leaving a final data set of 40 samples to analyse with TMT-B. In addition, a total of 4 samples from participant 1 and 8 were excluded as outliers, leaving a final data set of 38 samples to analyse with TMT-A.

One of the key objectives in this study was to examine the degree to which association exists between mobile gameplay behaviour and cognitive abilities. Spearman's rank correlation coefficients were computed to assess the relationship between pairs of variables. For ease of reading, the acronyms in Table 4 are used throughout the rest of the paper.

\subsection{Touch Data}

6.1.1 The Number of Touch Interactions and Cognitive Performance. In Tetris, as shown in Table 5, MEM was significantly correlated with the total number of leftward swipes $(\mathrm{r}=.41, \mathrm{p}<.01)$, the total number of horizontal swipes $(\mathrm{r}=.39, \mathrm{p}=.01)$, and the total number of swipes $(\mathrm{r}=.31, \mathrm{p}<.05)$. The results indicate that increases in the total number of touch interactions are correlated with increases in MEM. In addition, VISP showed a significant 
Table 3. Extracted gameplay features for each game.

\begin{tabular}{ll}
\hline Feature & Description \\
\hline Tetris & \\
\hline Max score & The maximum score achieved in the entire session \\
Max total taps per shape* & The maximum number of taps \\
Mean total taps per shape* & The average number of taps \\
Max total swipes per shape* & The maximum number of swipes \\
Mean total swipes per shape* & The average number of swipes \\
Max total interactions per shape* & The maximum number of taps and swipes \\
Mean total interactions per shape* & The average number of taps and swipes \\
Max rows completed per shape* & The maximum number of rows completed \\
Mean rows completed per shape* & The average number of rows completed \\
\hline Fruit Ninja & \\
\hline Max score & The maximum score achieved in the entire session \\
Mean overall air time & The average time a ball was in the air including the missing balls \\
Mean air time before being cut & The average time a ball was in the air before being cut \\
Max air time before being cut & The maximum time a ball was in the air before being cut \\
Min air time before being cut & The minimum time a ball was in the air before being cut \\
Mean cut position $x$ & The average cut position in x axis \\
Mean cut position y & The average cut position in y axis \\
\hline Candy Crush & \\
\hline Max level & The maximum level reached in the entire session \\
Max score & The maximum score achieved in the entire sesion \\
Max score in level** & The maximum score achieved in each level \\
*Based on actions performed before a single shape fell into the stack \\
**Level 1, 2, 3 and 4
\end{tabular}

Table 4. Acronym Table.

\begin{tabular}{llccc}
\hline Acronym & Description & Tetris & Fruit Ninja & Candy Crush \\
\hline ATN & Score from attention subtasks in ACE-III & $\checkmark$ & $\checkmark$ & $\checkmark$ \\
MEM & Score from memory subtasks in ACE-III & $\checkmark$ & & \\
VISP & Score from visuospatial subtasks in ACE-III & $\checkmark$ & $\checkmark$ & \\
TMTA & Time spent completing the task in TMT part A & $\checkmark$ & $\checkmark$ & $\checkmark$ \\
TMTB & Time spent completing the task in TMT part B & $\checkmark$ & $\checkmark$ & $\checkmark$ \\
RESIN & Response inhibition in Stroop test & & $\checkmark$ & $\checkmark$ \\
\hline
\end{tabular}

correlation with the total number of downward swipes $(\mathrm{r}=.40, \mathrm{p}<.01)$ and the total number of swipes $(\mathrm{r}=.31, \mathrm{p}<.05)$.

6.1.2 Swipe Length and Cognitive Performance. In Tetris, there was a positive correlation between ATN and the mean length of leftward swipes $(\mathrm{r}=.385, \mathrm{p}<.05)$. However, no significant correlation was found between rightward 
Table 5. Correlation between each touch feature and each cognitive performance in Tetris.

\begin{tabular}{lccccl}
\hline Feature & ATN & MEM & VISP & TMTA & TMTB \\
\hline Total Number of Rightward Swipes & 0.131 & 0.287 & 0.243 & $0.404 *$ & 0.177 \\
Total Number of Leftward Swipes & 0.086 & $0.410 * *$ & 0.267 & 0.117 & 0.058 \\
Total Number of Horizontal Swipes & 0.142 & $0.386 * *$ & 0.266 & 0.264 & 0.122 \\
Total Number of Downward Swipes & 0.105 & 0.119 & $0.404 * *$ & 0.123 & 0.245 \\
Total Number of Swipes & 0.129 & $0.305 *$ & $0.313 *$ & 0.252 & 0.191 \\
Total Number of Taps & -0.005 & 0.278 & 0.286 & -0.021 & 0.195 \\
Mean Length of Rightward Swipes & 0.100 & -0.010 & 0.079 & $0.336 *$ & $0.442 * *$ \\
Mean Length of Leftward Swipes & $0.385 *$ & 0.164 & 0.162 & $0.305 !$ & $0.462 * *$ \\
Mean Length of Horizontal Swipes & 0.266 & 0.102 & 0.157 & $0.319 !$ & $0.486 * *$ \\
Mean Length of Downward Swipes & 0.025 & -0.271 & 0.044 & 0.216 & 0.148 \\
Mean Length of Swipes & 0.173 & -0.052 & 0.115 & $0.356 *$ & $0.441 * *$ \\
Mean Speed of Rightward Swipes & 0.195 & 0.114 & 0.148 & $0.367 *$ & $0.347 *$ \\
Mean Speed of Leftward Swipes & $0.379 *$ & $0.314 *$ & 0.206 & $0.358 *$ & 0.291 \\
Mean Speed of Horizontal Swipes & $0.311 *$ & 0.227 & 0.182 & $0.429 * *$ & $0.364 *$ \\
Mean Speed of Downward Swipes & 0.080 & -0.184 & 0.100 & $0.352 *$ & 0.090 \\
Mean Speed of Swipes & 0.268 & 0.113 & 0.246 & $0.425 * *$ & 0.256 \\
\hline
\end{tabular}

${ }^{*} \mathrm{p}<.05,{ }^{* *} \mathrm{p}<.01, ! \mathrm{p}<=.06$

[See acronym description in Table 4]

Table 6. Correlation between each touch feature and each cognitive performance in Fruit Ninja.

\begin{tabular}{lrcccl}
\hline Feature & ATN & VISP & TMTA & TMTB & RESIN \\
\hline Mean Length of Swipes & 0.018 & -0.288 & 0.149 & 0.180 & $0.312 *$ \\
Mean Speed of Swipes & -0.071 & $-0.323 *$ & 0.092 & 0.083 & $0.530 * * *$ \\
Mean Directness Index of Swipes & -0.045 & -0.267 & 0.132 & 0.244 & $0.301 *$ \\
\hline
\end{tabular}

${ }^{*} \mathrm{p}<.05,{ }^{* *} \mathrm{p}<.01,{ }^{* * *} \mathrm{p}<=.001$

[See acronym description in Table 4]

swipes and ATN ( $p>.05)$. TMTB was significantly correlated with the mean length of rightward swipes $(\mathrm{r}=.44$, $\mathrm{p}<.01)$, leftward swipes $(\mathrm{r}=.46, \mathrm{p}<.01)$, horizontal swipes $(\mathrm{r}=.49, \mathrm{p}=.001)$ and all swipes $(\mathrm{r}=.44, \mathrm{p}<.01)$. Coherent results were found in TMTA as it showed a significant correlation with the mean length of rightward swipes $(\mathrm{r}$ $=.34, \mathrm{p}<.05)$ and all swipes $(\mathrm{r}=.36, \mathrm{p}<.05)$. It is worth noting that there were positive correlations between TMTA and the mean length of left swipes $(r=.31, p=.06)$ and horizontal swipes $(r=.32, p=.051)$, though, they were not statistically significant as the p-values were greater than .05 . Similar results were found in Candy Crush, where TMTB was significantly correlated with the mean length of downward swipes $(\mathrm{r}=.34, \mathrm{p}<.05)$ and vertical swipes $(\mathrm{r}=.35, \mathrm{p}<.05)$. The results imply that increases in the swipe length were correlated with increases in the time spent completing the TMT tasks. Furthermore, in Candy Crush, RESIN was significantly correlated with the mean length of rightward swipes $(r=.34, \mathrm{p}<.01)$, downward swipes $(\mathrm{r}=.47, \mathrm{p}<.01)$, leftward swipes $(\mathrm{r}=.33, \mathrm{p}<.05)$, horizontal swipes $(\mathrm{r}=.39, \mathrm{p}<.01)$, vertical swipes $(\mathrm{r}=.41, \mathrm{p}<.01)$ and all swipes $(\mathrm{r}=.43, \mathrm{p}<.01)$. A coherent result was found in Fruit Ninja as RESIN was significantly correlated with the mean length of swipes $(r=.31, p<.05)$. 
Table 7. Correlation between each touch feature and each cognitive performance in Candy Crush.

\begin{tabular}{lrrrr}
\hline Feature & ATN & TMTA & TMTB & RESIN \\
\hline Total Number of Rightward Swipes & -0.188 & -0.173 & 0.138 & 0.123 \\
Total Number of Leftward Swipes & -0.102 & -0.164 & -0.001 & -0.111 \\
Total Number of Horizontal Swipes & -0.203 & -0.261 & 0.013 & -0.012 \\
Total Number of Upward Swipes & 0.212 & 0.020 & 0.068 & -0.034 \\
Total Number of Downward Swipes & -0.278 & -0.198 & -0.030 & 0.105 \\
Total Number of Vertical Swipes & -0.160 & -0.187 & -0.060 & 0.093 \\
Total Number of Swipes & -0.189 & -0.203 & 0.065 & 0.019 \\
Mean Length of Rightward Swipes & 0.173 & -0.165 & 0.145 & $0.336 *$ \\
Mean Length of Leftward Swipes & 0.190 & -0.151 & 0.171 & $0.333 *$ \\
Mean Length of Horizontal Swipes & 0.184 & -0.154 & 0.148 & $0.393 * *$ \\
Mean Length of Upward Swipes & 0.129 & -0.206 & 0.229 & 0.208 \\
Mean Length of Downward Swipes & 0.137 & -0.085 & $0.336 *$ & $0.472 * *$ \\
Mean Length of Vertical Swipes & 0.185 & -0.167 & $0.345 *$ & $0.413 * *$ \\
Mean Length of Swipes & 0.190 & -0.157 & 0.265 & $0.429 * *$ \\
Mean Speed of Rightward Swipes & 0.154 & -0.165 & 0.122 & $0.346 *$ \\
Mean Speed of Leftward Swipes & 0.174 & -0.135 & 0.092 & $0.339 *$ \\
Mean Speed of Horizontal Swipes & 0.148 & -0.157 & 0.107 & $0.402 * *$ \\
Mean Speed of Upward Swipes & 0.115 & -0.070 & 0.257 & 0.282 \\
Mean Speed of Downward Swipes & 0.144 & -0.074 & 0.219 & $0.404 * *$ \\
Mean Speed of Vertical Swipes & 0.148 & -0.077 & 0.267 & $0.365 *$ \\
Mean Speed of Swipes & 0.169 & -0.094 & 0.188 & $0.372 *$ \\
\hline
\end{tabular}

${ }^{*} \mathrm{p}<.05,{ }^{* *} \mathrm{p}<.01$

[See acronym description in Table 4]

6.1.3 Swipe Speed and Cognitive Performance. In Tetris, TMTA was significantly correlated with the mean speed of rightward swipes $(\mathrm{r}=.37, \mathrm{p}<.05)$, downward swipes $(\mathrm{r}=.35, \mathrm{p}<.05)$, leftward swipes $(\mathrm{r}=.36, \mathrm{p}<.05)$, horizontal swipes $(\mathrm{r}=.43, \mathrm{p}<.01)$ and all swipes $(\mathrm{r}=.43, \mathrm{p}<.01)$. Similar results were found in TMTB as it showed a significant correlation with the mean speed of rightward swipes $(\mathrm{r}=.35, \mathrm{p}<.05)$ and horizontal swipes $(\mathrm{r}=.36, \mathrm{p}<.05)$. The results indicate that increases in the swipe speed were correlated with increases in the time spent completing TMT tasks. Furthermore, the mean speed of leftward swipes was significantly correlated with the ACE-III subtasks; $\operatorname{ATN}(\mathrm{r}=.38, \mathrm{p}<.01)$ and MEM $(\mathrm{r}=.31, \mathrm{p}<.05)$. Interestingly, the mean swipe speed in Fruit Ninja was negatively correlated with VISP $(\mathrm{r}=-.32, \mathrm{p}<.05)$. The mean swipe speed showed a significant correlation with RESIN $(\mathrm{r}=.53$, $\mathrm{p}<.001)$. Coherent results were found in Candy Crush as RESIN was significantly correlated with the mean speed of rightward swipes $(\mathrm{r}=.35, \mathrm{p}<.05)$, downward swipes $(\mathrm{r}=.40, \mathrm{p}<.01)$, leftward swipes $(\mathrm{r}=.34, \mathrm{p}<.05)$, horizontal swipes $(\mathrm{r}=.40, \mathrm{p}<.01)$, vertical swipes $(\mathrm{r}=.37, \mathrm{p}<.05)$ and all swipes $(\mathrm{r}=.37, \mathrm{p}<.05)$.

6.1.4 Directness Index and Cognitive Performance. In Fruit Ninja, there was a positive correlation between the mean directness index of swipes and RESIN $(r=.30, p<.05)$. The result indicates that increases in the directness index of swipes were correlated with decreases in response inhibition ability. 
Table 8. Correlation between each sensor feature and each cognitive performance in Tetris.

\begin{tabular}{lrrrcr}
\hline Feature & ATN & MEM & VISP & TMTA & TMTB \\
\hline Mean Acceleration Magnitude & 0.174 & -0.036 & 0.035 & 0.128 & 0.047 \\
Sum Acceleration Magnitude & 0.138 & 0.073 & 0.265 & $-0.419 * *$ & -0.047 \\
SD Acceleration on X axis & -0.018 & 0.025 & -0.219 & -0.124 & -0.202 \\
SD Acceleration on Y axis & 0.000 & 0.052 & 0.044 & 0.052 & 0.064 \\
SD Acceleration on Z axis & -0.103 & 0.115 & -0.142 & 0.188 & 0.245 \\
SD Acceleration Magnitude & -0.186 & -0.010 & -0.158 & 0.280 & 0.141 \\
Mean Rotational Speed Magnitude & 0.013 & 0.105 & -0.030 & 0.174 & 0.032 \\
Sum Rotational Speed Magnitude & 0.023 & 0.110 & -0.007 & 0.144 & 0.041 \\
SD Rotational Speed around X axis & -0.026 & 0.203 & -0.046 & $0.405 *$ & 0.179 \\
SD Rotational Speed around Y axis & 0.109 & 0.045 & -0.067 & 0.134 & 0.071 \\
SD Rotational Speed around Z axis & 0.081 & -0.080 & 0.062 & 0.206 & 0.135 \\
SD Rotational Speed Magnitude & 0.048 & 0.044 & -0.079 & 0.287 & 0.132 \\
\hline
\end{tabular}

${ }^{*} \mathrm{p}<.05,{ }^{* *} \mathrm{p}<.01$

[See acronym description in Table 4]

Table 9. Correlation between each sensor feature and each cognitive performance in FruitNinja.

\begin{tabular}{lrrrrr}
\hline Feature & ATN & VISP & TMTA & TMTB & RESIN \\
\hline Mean Acceleration Magnitude & -0.060 & -0.014 & -0.068 & 0.183 & 0.205 \\
Sum Acceleration Magnitude & -0.224 & -0.010 & 0.099 & -0.194 & -0.081 \\
SD Acceleration on X axis & 0.032 & -0.118 & -0.141 & 0.064 & 0.112 \\
SD Acceleration on Y axis & -0.128 & -0.238 & $-0.357 *$ & 0.032 & 0.213 \\
SD Acceleration on Z axis & -0.068 & -0.130 & $-0.352 *$ & 0.085 & 0.225 \\
SD Acceleration Magnitude & -0.169 & -0.097 & -0.026 & 0.229 & 0.266 \\
Mean Rotational Speed Magnitude & 0.000 & -0.175 & 0.112 & $0.312 *$ & 0.286 \\
Sum Rotational Speed Magnitude & -0.069 & -0.215 & 0.102 & 0.269 & 0.293 \\
SD Rotational Speed around X axis & -0.034 & -0.142 & 0.194 & $0.491 * *$ & 0.190 \\
SD Rotational Speed around Y axis & -0.120 & -0.164 & 0.070 & 0.198 & 0.268 \\
SD Rotational Speed around Z axis & -0.081 & -0.277 & 0.136 & $0.336 *$ & 0.300 \\
SD Rotational Speed Magnitude & -0.141 & -0.163 & 0.087 & $0.347 *$ & 0.263 \\
\hline
\end{tabular}

${ }^{*} \mathrm{p}<.05,{ }^{* *} \mathrm{p}<.01$

[See acronym description in Table 4]

\subsection{Sensor Data}

6.2.1 Device Motion and Cognitive Performance. In Tetris, the sum acceleration magnitude showed a moderate negative correlation with TMTA $(\mathrm{r}=-.42, \mathrm{p}<.01)$. In contrast to Candy Crush, the sum acceleration magnitude was positively correlated with TMTA $(r=.33, p<.05)$ and no significant correlation was found with this feature in Fruit Ninja. The SD acceleration on the $y$ and $z$ axes in Fruit Ninja showed negative correlations with TMTA ( $r=-.36$, $\mathrm{p}<.05$ and $\mathrm{r}=-.35, \mathrm{p}<.05$, respectively). However, no significant correlation was found with the features related to 
Table 10. Correlation between each sensor feature and each cognitive performance in Candy Crush.

\begin{tabular}{lrcrr}
\hline Feature & ATN & TMTA & TMTB & RESIN \\
\hline Mean Acceleration Magnitude & 0.118 & -0.007 & -0.026 & -0.226 \\
Sum Acceleration Magnitude & 0.229 & $0.326 *$ & 0.071 & -0.097 \\
SD Acceleration on X axis & -0.195 & -0.227 & -0.120 & 0.079 \\
SD Acceleration on Y axis & 0.123 & -0.094 & -0.017 & 0.194 \\
SD Acceleration on Z axis & -0.004 & -0.033 & 0.200 & 0.210 \\
SD Acceleration Magnitude & -0.202 & 0.121 & 0.213 & 0.111 \\
Mean Rotational Speed Magnitude & -0.171 & 0.132 & 0.164 & -0.046 \\
Sum Rotational Speed Magnitude & -0.158 & 0.167 & 0.161 & -0.042 \\
SD Rotational Speed around X axis & -0.129 & $0.335 *$ & 0.273 & 0.040 \\
SD Rotational Speed around Y axis & -0.146 & 0.124 & 0.188 & 0.073 \\
SD Rotational Speed around Z axis & 0.005 & -0.107 & 0.157 & 0.257 \\
SD Rotational Speed Magnitude & -0.123 & 0.173 & 0.250 & 0.181 \\
\hline
\end{tabular}
${ }^{*} \mathrm{p}<.05,{ }^{* *} \mathrm{p}<.01$

[See acronym description in Table 4]

the SD acceleration in Candy Crush. The SD rotational speed around the $\mathrm{x}$-axis was significantly correlated with TMTA in both Tetris $(\mathrm{r}=.41, \mathrm{p}=.01)$ and Candy Crush $(\mathrm{r}=.34, \mathrm{p}=.04)$.

In Fruit Ninja, TMTB was significantly correlated with the mean rotational speed magnitude $(r=.31, p<.05)$, the $\mathrm{SD}$ rotational speed around the $\mathrm{x}$-axis $(\mathrm{r}=.49, \mathrm{p}<.01)$, the $\mathrm{SD}$ rotational around the $\mathrm{y}$-axis $(\mathrm{r}=.34, \mathrm{p}<.05)$ and the SD rotational magnitude $(\mathrm{r}=.35, \mathrm{p}<.05)$.

\subsection{Gameplay}

6.3.1 Max Score and Cognitive Performance. In Tetris, the max score was significantly correlated with VISP $(\mathrm{r}=.32, \mathrm{p}<.05)$. Although no significant correlation was found with the max score in Candy Crush, the max score in level 3 showed a weak negative correlation with TMTA $(\mathrm{r}=-.38, \mathrm{p}<.05)$. Overall, increases in max scores in the games were correlated with increases in cognitive performance in certain domains.

6.3.2 Game Response Time and Cognitive Performance. In Fruit Ninja, the mean air time before being cut was significantly correlated with VISP $(\mathrm{r}=.33, \mathrm{p}<.05)$ and RESIN $(\mathrm{r}=-.31, \mathrm{p}<.05)$. It implies that increases in mean air time before being cut were correlated with increases in performance on visuospatial function and response inhibition.

6.3.3 Interaction Area and Cognitive Performance. In Fruit Ninja, the mean cut position in y-axis was significantly correlated with ATN $(\mathrm{r}=.39, \mathrm{p}=.01)$ and VISP $(\mathrm{r}=.5, \mathrm{p}<.001)$. These results imply that increases in the height of the interaction area on the screen were correlated with increases in cognitive performance in certain domains. The higher users interact on the screen, the better the cognitive performance. Furthermore, the mean cut position in $\mathrm{x}$-axis was significantly correlated with VISP $(\mathrm{r}=.42, \mathrm{p}=<.01)$. It indicates that increases in horizontal positions users interact on the screen were associated with increases in performance in visuospatial abilities.

\section{DISCUSSION}

This study investigates the possible use of popular mobile games with a strong track record in user engagement and re-playability to assess cognitive function. We looked at whether cognitive performance can be predicted 
from gameplay behaviour, including swipe patterns, sensor data and in-game metrics. In this study, we present exploratory results of the relationship between mobile gameplay behavioural data and player's cognitive performance. We acknowledge that the analysis was evaluated with a relatively small number of healthy participants. However, our analysis has shown statistically significant results (with p-value $<.01$ in some cases) that provide compelling evidence to support the presence of the correlations.

\subsection{Addressing Research Questions}

7.1.1 RQ1: Are the swipe length and shape of touch gestures related to changes in cognitive performance? and RQ2: Is the speed of touch gestures related to changes in cognitive performance? We found that overall swipe speed features were positively correlated with TMTA and TMTB in Tetris and Candy Crush, while overall swipe speed features in Fruit Ninja and Candy Crush were positively correlated with RESIN. These results imply that increases in swipe speed were associated with decreases in performance on these cognitive functions (visual search, mental flexibility and response inhibition). This finding demonstrates a clear diversion from the studies on traditional handwriting movement $[28,36,44]$. Indeed, in handwriting studies, higher hand movement speed is correlated with increase in cognitive performance. However, we consider that the seemingly contradictory finding can be explained by the significant differences in user intention, between handwriting and gameplay. Based on gameplay observations, we noted that fast and erratic gestures in games like Fruit Ninja, tend to occur when players narrowly miss certain objects that they are expected to interact with, within a limited time frame. We therefore hypothesise that the temporal nature of game interaction can lead to more erratic and fast gestures, as the cognitive abilities of the players decline. The positive correlation between the directness index of swipes and response inhibition ability as well as the swipe patterns idiosyncratic to Fruit Ninja provide evidence to support this explanation. It is consistent with the negative correlation we identify between VISP and swipe speed in Fruit Ninja. The results seem to suggest that these fast swipes, demonstrate decreases in visuospatial abilities, and are indeed rushed movements.

Another interesting finding is that swipe length related features were positively and significantly correlated with TMTA, TMTB and RESIN. In other words, it indicates that increase in swipe length is associated with decreased performance in visual search, mental flexibility and response inhibition. Hence, swipe length could be useful features to be included in game design for measuring these cognitive functions. Interestingly, we found significant and strong correlations between the mean swipe speeds and the mean swipe lengths in the corresponding directions (all $\mathrm{r}>.8, \mathrm{p}<.01$ in Tetris, $\mathrm{r}>.74, \mathrm{p}<.01$ in Candy Crush and $\mathrm{r}=.44, \mathrm{p}<.01$ in Fruit Ninja). This can imply that faster swipes were correlated with longer swipes. When further investigating the correlations found in Tetris, we observe significant relations between touch features (length and speed) in the leftward direction and attention capability, but no correlation was found between the rightward gestural features and attentional ability. We believe that this discrepancy is related to the strong attentional bias of left hemisphere individuals to stimuli on the right visual field [40] that may potentially influence the gesture patterns and our correlation results. Anecdotal observational evidence indicates a tendency on Tetris players to stack most of the blocks on the left side of the screen while leaving the right side empty for straight line blocks. This tendency can be explained by the attention bias for right-handed individuals, however, we don't have sufficient evidence to demonstrate such a link. Nevertheless, we feel that results like these are strong indicators that future research should examine the influence of this attentional bias of the right-handed users on gestural characteristics.

7.1.2 RQ3: Are the characteristics of the physical movement of the mobile device related to changes in cognitive performance? The bivariate analysis of sensor data provides evidence showing that device acceleration and rotational movement were associated with certain cognitive abilities, specifically visual search, attention and mental flexibility. The rotational speed-related features showed positive correlations with TMTB, particularly in Fruit Ninja. We argue that this may be due to the eccentric finger stroke behaviour that generates a greater 
degree of device motion and rotation than in the other two games. This implication was consistent with the positive correlations between SD acceleration in the $\mathrm{z}$-axis and the mean swipe speed $(\mathrm{r}=.467, \mathrm{p}<.01)$ and between gyro-related features and the mean swipe speed ( $\mathrm{r}$ between .44 and .54 , all with $\mathrm{p}<.01$ ). Although these findings are to be taken cautiously (as only some evidence was found in Tetris and Candy Crush), a greater degree of rotational movement around $\mathrm{x}$ axis is positively associated with TMTA (lower performance on visual search and attention). This seems to be coherent with the aforementioned findings in touch-based features that more rapid and active interactions with the device correspond to poorer performance on TMTA. Particularly, faster swipes in Candy Crush exhibited an association with poorer inhibition control measured by RESIN. It seems reasonable to conclude that these fast swipes were carried out less deliberately due to an instinctive response. Nonetheless, the SD rotational speed around the $\mathrm{x}$-axis demonstrated a negative correlation with the performance measured by TMTA. This device motion feature was significantly correlated with decreases in swipe speed (all with $\mathrm{r}<-.35$, $\mathrm{p}<.05$ except the mean speed of leftward swipes). In other words, slower swipes were correlated with a higher degree of rotational movement variability around the $\mathrm{x}$-axis and therefore decreases in cognitive performance measured by TMTA. These correlation results suggest that the significance between these device movement variability features and cognitive performance found in Candy Crush were not influenced by the rapid screen interaction as initially speculated. Instead, it appears that these significant correlations were mainly influenced by the device motion alone when there was no screen interaction. This implication was consistent with the findings in Tetris as no significant correlation was found between touch and motion sensor features. As the observed correlations seem to be dependent on game mechanics, it remains unclear whether these device motion features alone can be used as proxy markers for changes in cognitive performance.

Similarly, a mixed picture emerges with the acceleration-related features. In Tetris higher magnitude accelerations are negatively correlated with TMTA (better attention and visual search capabilities), while there is weak evidence that the opposite is the case in Candy Crush. One possible explanation may be that device orientation plausibly reflects the level of attention in the games but this orientation might also be influenced by the jittery motion from touch interaction. Further research would be required to examine this assumption.

7.1.3 Game Performance Patterns. Unsurprisingly, in-game scoring features demonstrate an association with cognitive performance. A significant correlation between max score and visuospatial abilities in Tetris suggests that high demands are placed on visuospatial working memory when rotating and moving blocks to complete a row. Our finding supports the recommendation of Tetris as a visuospatial diagnostic task from previous studies $[13,16]$. Contrary to our expectation, increases in the mean time to cut items in Fruit Ninja showed a positive correlation with increases in visuospatial abilities. We believe this is due to the fact that since multiple targets often appear simultaneously on the screen, it can require more than one swipe not to miss any items. As a result, higher performing players may execute a second swipe to locate and slice an item that almost fell off the screen, while lower performing players do not. Taking this into account, the mean airtime before cutting therefore increased for higher performing players.

\subsection{Game Mechanics, Cognitive Demand and Gestural Characteristics}

It is worth noting that swipe gesture features only show significant correlations with particular cognitive capacities in particular games. For instance, the mean speed of horizontal swipes was significantly associated with ATN, TMTA and TMTB in Tetris. Conversely, we did not find such a relationship in Candy Crush. We argue that the differences in the swipe characteristics among these games are potentially influenced by the game mechanics and related cognitive demand of the given game tasks. In particular, Tetris demands attention and visuospatial functions to determine the spatial relations to complete a row of blocks whereas Candy Crush requires visual search capacity to locate and make a move to create a possible match of items. A related point to consider is that in contrast to the positive correlation with ATN, faster speed in horizontal swipes in Tetris was 
correlated with decreases in visual search abilities measured by TMT tasks. The most plausible explanation is that a significant demand on visual spatial attention weakens visual search performance in Tetris [51]. Yet another possible explanation is that the underlying game objective of gestural interactions may have some influence on swipe characteristics [5]. We indeed found that there was no difference between the speed of horizontal and vertical swipes in Candy Crush $[\mathrm{M}=-6.69, \mathrm{t}=-.181, \mathrm{p}=.857]$ whereas the speed of horizontal swipes was significantly different from downward swipes in Tetris [M=-996.64, $\mathrm{t}=-8.86, \mathrm{p}<.001]$. It could be explained that in Candy Crush, swipes in all four directions were solely to swap item positions resulting in no speed difference in these gesture directions. Conversely in Tetris, with the intention to force the block to fall as quickly as possible, downward swipes were carried out in much faster speed comparing to other swipe directions. In addition, the number of downward swipes demonstrated a significant correlation with VISP. These results support our speculation that in Tetris features in downward direction could indicate players' confidence in the target location reflecting better performance in visuospatial capacity. Taken together, these observations seem to point to the possibility that gestural characteristics are likely to be influenced by game mechanics and respective cognitive demand.

\subsection{Game Design Recommendations}

Based on the discussion outlined above, we can offer a number of recommendations and considerations for designing a practical game-based cognitive assessment instrument. Firstly, we should point out that game based cognitive assessment cannot be considered as a direct replacement for traditional, paper-based, assessment instruments. Instead, we consider that the role of a game-based cognitive assessment instrument is primarily to provide means for more frequent and informal capture of changes in cognitive abilities over time, indicating the need for a more formal assessment when a significant and sustainable decline in cognitive abilities is observed. In that respect, such techniques could, for example, be used to assist in the long-term observation of users with dementia, or other conditions that affect cognitive abilities.

Secondly, in designing games to help assess cognitive performance, game mechanics can be designed to target specific cognitive abilities. For instance, assessing visuospatial functions would be possible within games where the user is expected to identify positions, orientations and shapes of visual stimuli relative to the game environment in space. Furthermore, game mechanics should involve user gestures that are triggered by such visual stimuli. These features would allow the capture of such gestures and explore features such as length, shape and orientation of gesture to discover changes in visuospatial abilities. These game mechanics can be found in shape matching games like Tetris or more traditional Shape Sorter games. Augmenting such games with time constraints is also a feature that can help capture changes in the player's processing speed and response to visual stimuli. As shown in this study, in time constraint games, faster gestures are correlated with reduced cognitive abilities, indicating potentially slow response to visual stimuli.

Furthermore, the underlying game objectives of performing specific gestures to manipulate visual stimuli should be linked to cognitive demand reflecting the player's ability to understand spatial relations. For example, tapping the screen to rotate an object or swiping down to drop an object with the correct orientation into the correct gap in Tetris. Specifically, the latter swipe down gesture in Tetris can demonstrate high confidence in identifying spatial relations between the presented stimuli and the game environment as demonstrated in our findings. More generally, within the mechanics of any game, certain gestures can potentially play that role of demonstrating the players' confidence in understanding and responding to visual stimuli in the game. These gestures can be exploited in the same way as the vertical gesture in Tetris, to identify correlations with cognitive abilities.

Lastly, it is important to consider that games that have been considered in this study tend to put a significant cognitive demand on the users. We believe that this is a feature that helps identify relations between game interaction features and cognitive abilities. Indeed, games that require intensive touch interactions, such as taps 
and swipes within a time constraint game duration, can help generate significant gesture data points, which subsequently can help identify changes in cognitive abilities. However, it should be considered that the intensity and cognitive demand by a game could potentially hinder user retention. Indeed, finding the right balance in the level of game intensity and user satisfaction is a broader challenge for the design of any computer game.

\subsection{Limitations and Future Directions}

Based on the presence of the relationships we found here, we believe there is potential in exploring machine learning techniques to examine the predictive capability of these extracted features for cognitive assessments. Furthermore, it is a question of future research to investigate whether user-game interaction behaviour can be used to identify patients with cognitive impairment and explore the long term changes in cognitive abilities for such individuals.

We should also acknowledge a number of limitations in our current study. According to the participant exclusion criteria, the results of our study are restricted to non-excessive gamers. Apart from the fatigue-inducing task, the in-game events, for instance, making wrong moves in the game, may also have an additive fatigue-inducing effect on the subsequent cognitive performance within the game. To minimise this influence on our measures we randomised game order and kept the gameplay duration on each game to a minimum. Moreover, in our experiment, data collection was under controlled lab conditions in that participants were asked to use only one finger to interact with the device at a time. This may not truly reflect their natural gameplay behaviour in a non-experimental environment. Future work should consider the potential effect of multi-touch gestures to validate the findings we drew from this study. Long-term studies in users' natural environment will also help shed light on the practicality of deploying such a system in the real world.

\section{CONCLUSIONS}

In this work, we have explored the feasibility of using gesture and movement data in mobile games to identify patterns that are associated with cognitive performance in cognitively healthy individuals. To the best of our knowledge, this is the first piece of work that investigates such links between patterns of touch interaction and device motions in these particular mobile games and cognitive performance. In particular, swipe speed and swipe length have been found to be significantly correlated with cognitive performance in visual search, mental flexibility and response inhibition. Collectively, these results appear consistent with the significant correlations found between swipe speed and swipe length. With regard to device motion features, our results have provided supporting evidence consistent with previous studies $[19,36,44]$ that individuals with poorer cognitive abilities tend to demonstrate a higher degree of phone movement variability when playing games. These findings show promising potential in using such features for game-based cognitive assessment. Our work relied on the use of existing popular games to demonstrate these effects. This is a strong indicator that our findings can be applied or incorporated in a range of existing games with a strong track record in user engagement. We believe that the results of this work will provide valuable insights for researchers in game interaction design to maximise the use of touch and device motion features with respect to cognitive assessment.

\section{ACKNOWLEDGMENTS}

This work was granted by the Office of the Higher Education Commission. Jittrapol Intarasirisawat was supported by the Strategic Ph.D. scholarship for Frontier Research Networks.

\section{REFERENCES}

[1] Anna Anzulewicz, Krzysztof Sobota, and Jonathan T. Delafield-Butt. 2016. Toward the Autism Motor Signature: Gesture patterns during smart tablet gameplay identify children with autism. Scientific reports 6 (2016), 31107. 
[2] Russell A. Barkley. 1997. Behavioral inhibition, sustained attention, and executive functions: constructing a unifying theory of ADHD. Psychological bulletin 121, 1 (1997), 65.

[3] Jennifer H. Barnett, Andrew D. Blackwell, Barbara J. Sahakian, and Trevor W. Robbins. 2015. The paired associates learning (PAL) test: 30 years of CANTAB translational neuroscience from laboratory to bedside in dementia research. Springer, 449-474.

[4] Chris Bevan and DanaÃń Stanton Fraser. 2016. Different strokes for different folks? Revealing the physical characteristics of smartphone users from their swipe gestures. International fournal of Human-Computer Studies 88 (apr 2016), 51-61. https://doi.org/10.1016/j.ijhcs. 2016.01.001

[5] Gary Burnett, Elizabeth Crundall, David Large, Glyn Lawson, and Lee Skrypchuk. 2013. A study of unidirectional swipe gestures on in-vehicle touch screens. In Proceedings of the 5th International Conference on Automotive User Interfaces and Interactive Vehicular Applications. ACM, 22-29.

[6] Niamh Caprani, Noel E., and Cathal Gurri. 2012. Touch Screens for the Older User. In Assistive Technologies. InTech. https://doi.org/10. $5772 / 38302$

[7] A. Collie, D. Darby, and P. Maruff. 2001. Computerised cognitive assessment of athletes with sports related head injury. British journal of sports medicine 35, 5 (Oct 2001), 297-302. LR: 20140613; JID: 0432520; RF: 29; 2001/10/02 10:00 [pubmed]; 2002/01/05 10:01 [medline]; 2001/10/02 10:00 [entrez]; ppublish.

[8] Ian J. Deary and Geoff Der. 2005. Reaction Time, Age, and Cognitive Ability: Longitudinal Findings from Age 16 to 63 Years in Representative Population Samples. Aging, Neuropsychology, and Cognition 12, 2 (jun 2005), 187-215. https://doi.org/10.1080/13825580590969235

[9] Bruno Dubois, Alessandro Padovani, Philip Scheltens, Andrea Rossi, and Grazia Dell'Agnello. 2015. Timely Diagnosis for Alzheimer's Disease: A Literature Review on Benefits and Challenges. Fournal of Alzheimer's Disease 49, 3 (Oct 2015), 617-631. https://doi.org/10. 3233/JAD- 150692

[10] Eletha Flores, Gabriel Tobon, Ettore Cavallaro, Francesca I. Cavallaro, Joel C. Perry, and Thierry Keller. 2008. Improving patient motivation in game development for motor deficit rehabilitation. In Proceedings of the 2008 International Conference on Advances in Computer Entertainment Technology. ACM, 381-384.

[11] Luciano Gamberini, Francesco Martino, Bruno Seraglia, Anna Spagnolli, Malena Fabregat, Francisco Ibanez, Mariano Alcaniz, and Javier Montesa Andrés. 2009. Eldergames project: An innovative mixed reality table-top solution to preserve cognitive functions in elderly people. In Human System Interactions, 2009. HSI'09. 2nd Conference on. IEEE, 164-169.

[12] Yuan Gao, Nadia Bianchi-Berthouze, and Hongying Meng. 2012. What does touch tell us about emotions in touchscreen-based gameplay? ACM Transactions on Computer-Human Interaction (TOCHI) 19, 4 (2012), 31.

[13] C. Shawn Green and Daphne Bavelier. 2003. Action video game modifies visual selective attention. Nature 423, 6939 (2003), 534.

[14] Daniel F. Hermens, Jim Lagopoulos, Juliette Tobias-Webb, Tamara De Regt, Glenys Dore, Lisa Juckes, Noeline Latt, and Ian B. Hickie. 2013. Pathways to alcohol-induced brain impairment in young people: a review. Cortex 49, 1 (2013), 3-17.

[15] John R. Hodges. 2007. Cognitive assessment for clinicians. Oxford University Press.

[16] Emily A. Holmes, Ella L. James, Emma J. Kilford, and Catherine Deeprose. 2010. Key Steps in Developing a Cognitive Vaccine against Traumatic Flashbacks: Visuospatial Tetris versus Verbal Pub Quiz. PLoS ONE 5, 11 (nov 2010), e13706. https://doi.org/10.1371/journal. pone. 0013706

[17] Susan Homack and Cynthia A. Riccio. 2004. A meta-analysis of the sensitivity and specificity of the Stroop Color and Word Test with children. Archives of clinical Neuropsychology 19, 6 (2004), 725-743.

[18] Sameer Jauhar, E. Jane Marshall, and Iain D. Smith. 2014. Alcohol and cognitive impairment. Advances in psychiatric treatment 20, 5 (2014), 304-313.

[19] Caroline Ketcham and George Stelmach. 2004. Movement control in the older adult. Technology for Adaptive Aging (01 2004), 64-92.

[20] Alexandra M Kueider, Jeanine M Parisi, Alden L Gross, and George W Rebok. 2012. Computerized cognitive training with older adults: a systematic review. PloS one 7, 7 (2012), e40588. https://doi.org/10.1371/journal.pone.0040588

[21] Robert Langner, Michael B Steinborn, Anjan Chatterjee, Walter Sturm, and Klaus Willmes. 2010. Mental fatigue and temporal preparation in simple reaction-time performance. Acta psychologica 133, 1 (Jan 2010), 64-72. https://doi.org/10.1016/j.actpsy.2009.10.001

[22] Alex Lau-Zhu, Emily A. Holmes, Sally Butterfield, and Joni Holmes. 2017. Selective Association Between Tetris Game Play and Visuospatial Working Memory: A Preliminary Investigation. Applied Cognitive Psychology 31, 4 (jul 2017), 438-445. https://doi.org/10.1002/acp.3339

[23] Megan E. Lenehan, Mathew J. Summers, Nichole L. Saunders, Jeffery J. Summers, and James C. Vickers. 2016. Does the Cambridge Automated Neuropsychological Test Battery (CANTAB) distinguish between cognitive domains in healthy older adults? Assessment 23, 2 (2016), 163-172.

[24] Qian Liu, Xinyi Zhu, Albert Ziegler, and Jiannong Shi. 2015. The effects of inhibitory control training for preschoolers on reasoning ability and neural activity. Scientific Reports 5, 1 (sep 2015). https://doi.org/10.1038/srep14200

[25] Monica Luciana. 2003. Practitioner review: computerized assessment of neuropsychological function in children: clinical and research applications of the Cambridge Neuropsychological Testing Automated Battery (CANTAB). Fournal of Child Psychology and Psychiatry 44, 5 (2003), 649-663. 
[26] Jim Lumsden, Elizabeth A Edwards, Natalia S Lawrence, David Coyle, and Marcus R Munafò. 2016. Gamification of Cognitive Assessment and Cognitive Training: A Systematic Review of Applications and Efficacy. FMIR Serious Games 4, 2 (jul 2016 ), e11. https://doi.org/10.2196/games.5888

[27] V. Manera, P. D. Petit, A. Derreumaux, I. Orvieto, M. Romagnoli, G. Lyttle, R. David, and P. H. Robert. 2015. 'Kitchen and cooking,' a serious game for mild cognitive impairment and Alzheimer's disease: a pilot study. Frontiers in aging neuroscience 7 (Mar 17 2015), 24. https://doi.org/10.3389/fnagi.2015.00024[doi] LR: 20170220; JID: 101525824; OID: NLM: PMC4362400; OTO: NOTNLM; 2015/01/14 [received]; 2015/02/22 [accepted]; epublish.

[28] P Mavrogiorgou, R Mergl, P Tigges, J El Husseini, A SchrÃűter, G Juckel, M Zaudig, and U Hegerl. 2001. Kinematic analysis of handwriting movements in patients with obsessive-compulsive disorder. Journal of neurology, neurosurgery, and psychiatry 70, 5 (May 2001), 605-12.

[29] Ziad S. Nasreddine, Natalie A. Phillips, ValÃlrie BÃldirian, Simon Charbonneau, Victor Whitehead, Isabelle Collin, Jeffrey L. Cummings, and Howard Chertkow. 2005. The Montreal Cognitive Assessment, MoCA: a brief screening tool for mild cognitive impairment. fournal of the American Geriatrics Society 53, 4 (2005), 695-699.

[30] K. Onoda, T. Hamano, Y. Nabika, A. Aoyama, H. Takayoshi, T. Nakagawa, M. Ishihara, S. Mitaki, T. Yamaguchi, H. Oguro, K. Shiwaku, and S. Yamaguchi. 2013. Validation of a new mass screening tool for cognitive impairment: Cognitive Assessment for Dementia, iPad version. Clinical interventions in aging 8 (2013), 353-360. https://doi.org/10.2147/CIA.S42342[doi] LR: 20150427; JID: 101273480; OTO: NOTNLM; 2013/04/10 06:00 [entrez]; 2013/04/10 06:00 [pubmed]; 2013/11/05 06:00 [medline]; ppublish.

[31] R. C. Petersen, J.C. Stevens, M. Ganguli, E. G. Tangalos, J.L. Cummings, and S. T. DeKosky. 2001. Practice parameter: Early detection of dementia: Mild cognitive impairment (an evidence-based review): Report of the Quality Standards Subcommittee of the American Academy of Neurology. Neurology 56, 9 (may 2001), 1133-1142. https://doi.org/10.1212/wnl.56.9.1133

[32] Trevor W. Robbins, M. James, Adrian M. Owen, Barbara J. Sahakian, L. McInnes, and P. Rabbitt. 1994. Cambridge Neuropsychological Test Automated Battery (CANTAB): a factor analytic study of a large sample of normal elderly volunteers. Dementia and geriatric cognitive disorders 5, 5 (1994), 266-281.

[33] Philippe Robert, Alexandra König, Hélene Amieva, Sandrine Andrieu, François Bremond, Roger Bullock, Mathieu Ceccaldi, Bruno Dubois, Serge Gauthier, and Paul-Ariel Kenigsberg. 2014. Recommendations for the use of Serious Games in people with Alzheimer's Disease, related disorders and frailty. Frontiers in aging neuroscience 6 (2014), 54

[34] Ned Sacktor, Michael P. McDermott, Karen Marder, Giovanni Schifitto, Ola A. Selnes, Justin C. McArthur, Yaakov Stern, Steve Albert, Donna Palumbo, and Karl Kieburtz. 2002. HIV-associated cognitive impairment before and after the advent of combination therapy. Journal of neurovirology 8, 2 (2002), 136-142.

[35] Akane Sano and Rosalind W. Picard. 2013. Stress recognition using wearable sensors and mobile phones. In Affective Computing and Intelligent Interaction (ACII), 2013 Humaine Association Conference on. IEEE, 671-676.

[36] A. Schröter, R. Mergl, K. Bürger, H. Hampel, H.-J. Möller, and U. Hegerl. 2003. Kinematic Analysis of Handwriting Movements in Patients with Alzheimer's Disease, Mild Cognitive Impairment, Depression and Healthy Subjects. Dementia and Geriatric Cognitive Disorders 15 , 3 (2003), 132-142. https://doi.org/10.1159/000068484

[37] A. Schweiger, GM Doniger, T. Dwolatzky, D. Jaffe, and ES Simon. 2003. Reliability of a novel computerized neuropsychological battery for mild cognitive impairment. Acta Neuropsychologica 1, 4 (2003), 407-413.

[38] Yoshihito Shigihara, Masaaki Tanaka, Akira Ishii, Seiki Tajima, Etsuko Kanai, Masami Funakura, and Yasuyoshi Watanabe. 2013. Two different types of mental fatigue produce different styles of task performance. Neurology, Psychiatry and Brain Research 19, 1 (feb 2013), 5-11. https://doi.org/10.1016/j.npbr.2012.07.002

[39] F. C. Soares, T. C. de Oliveira, L. D. de Macedo, A. M. Tomas, D. L. Picanco-Diniz, J. Bento-Torres, N. V. Bento-Torres, and C. W. Picanco-Diniz. 2014. CANTAB object recognition and language tests to detect aging cognitive decline: an exploratory comparative study. Clinical interventions in aging 10 (Dec 19 2014), 37-48. https://doi.org/10.2147/CIA.S68186[doi] LR: 20170220; JID: 101273480; OID: NLM: PMC4279672; OTO: NOTNLM; epublish.

[40] Kevin M. Spencer and Marie T. Banich. 2005. Hemispheric biases and the control of visuospatial attention: an ERP study. BMC Neuroscience 6, 1 (24 Aug 2005), 51. https://doi.org/10.1186/1471-2202-6-51

[41] Statista. [n.d.]. U.S. average age of video gamers 2018 | Statistic. url: https://www.statista.com/statistics/189582/age-of-us-video-gameplayers-since-2010/. https://www.statista.com/statistics/189582/age- of-us-video-game-players-since-2010/ Accessed: 22-March-2019.

[42] Alvaro D. Taveira and Sang D. Choi. 2009. Review Study of Computer Input Devices and Older Users. International fournal of Human-Computer Interaction 25, 5 (jun 2009), 455-474. https://doi.org/10.1080/10447310902865040

[43] H. Gerry Taylor and Kenneth M. Heilman. 1980. Left-Hemisphere Motor Dominance in Righthandersi. Cortex 16, 4 (Dec. 1980), 587-603. https://doi.org/10.1016/s0010-9452(80)80006-2

[44] Peter Tigges, Roland Mergl, Thomas Frodl, Eva M. Meisenzahl, Jürgen Gallinat, Andreas Schröter, Michael Riedel, Norbert Müller, Hans-Jürgen Möller, and Ulrich Hegerl. 2000. Digitized analysis of abnormal hand-motor performance in schizophrenic patients. Schizophrenia Research 45, 1-2 (sep 2000), 133-143. https://doi.org/10.1016/s0920-9964(99)00185-1

[45] Tom N. Tombaugh. 2004. Trail Making Test A and B: normative data stratified by age and education. Archives of clinical neuropsychology 19, 2 (2004), 203-214.

, Vol. 1, No. 1, Article . Publication date: September 2019. 
[46] Tom N. Tombaugh and Nancy J. McIntyre. 1992. The miniâĂ Řmental state examination: a comprehensive review. Fournal of the American Geriatrics Society 40, 9 (1992), 922-935.

[47] T. Tong, M. Chignell, M. C. Tierney, and J. Lee. 2016. A Serious Game for Clinical Assessment of Cognitive Status: Validation Study. 7MIR serious games 4, 1 (May 27 2016), e7. https://doi.org/10.2196/games.5006[doi] LR: 20170220; JID: 101645255; OID: NLM: PMC4902858; OTO: NOTNLM; 2015/08/05 [received]; 2016/02/29 [accepted]; 2015/11/30 [revised]; epublish.

[48] Unity3D. [n.d.]. Match 3 Jelly Garden Complete Project Asset Store. url: https://assetstore.unity.com/packages/templates/systems/match3-jelly-garden-complete-project-43260. https://assetstore.unity.com/packages/templates/systems/match-3-jelly-garden-completeproject-43260 Accessed: 11-July-2018.

[49] Unity3D. [n.d.]. Object/Ball Slicing Example Game Unity3D Asset Store. url: https://assetstore.unity.com/packages/templates/objectball-slicing-example-game-76330. https://assetstore.unity.com/packages/templates/object-ball-slicing-example-game-76330 Accessed: 11-July-2018.

[50] Unity3D. [n.d.]. Tetris Template Mobile Ready Unity3D Asset Store. url: https://assetstore.unity.com/packages/templates/tetris-templatemobile-ready-72717. https://assetstore.unity.com/packages/templates/tetris-template-mobile-ready-72717 Accessed: 11-July-2018.

[51] Geoffrey F. Woodman and Steven J. Luck. 2004. Visual search is slowed when visuospatial working memory is occupied. Psychonomic bulletin \& review 11, 2 (2004), 269-274.

[52] Anthony S. Zigmond and R. Philip Snaith. 1983. The hospital anxiety and depression scale. Acta Psychiatrica Scandinavica 67, 6 (1983), 361-370.

[53] Gokhan Zorluoglu, Mustafa E. Kamasak, Leyla Tavacioglu, and Pinar O. Ozanar. 2015. A mobile application for cognitive screening of dementia. Computer methods and programs in biomedicine 118, 2 (2015), 252-262.

[54] Stelios Zygouris, Dimitrios Giakoumis, Konstantinos Votis, Stefanos Doumpoulakis, Konstantinos Ntovas, Sofia Segkouli, Charalampos Karagiannidis, Dimitrios Tzovaras, and Magda Tsolaki. 2015. Can a virtual reality cognitive training application fulfill a dual role? Using the virtual supermarket cognitive training application as a screening tool for mild cognitive impairment. Fournal of Alzheimer's Disease 44, 4 (2015), 1333-1347.

[55] Stelios Zygouris and Magda Tsolaki. 2015. Computerized cognitive testing for older adults: a review. American fournal of Alzheimer's Disease \& Other Dementias ${ }^{\circledR}$ 30, 1 (2015), 13-28. 